\title{
Three-dimensional Cell Culture Technique and Pathophysiology
}

\author{
Michiya Matsusaki ${ }^{1}$, Charles Patrick Case ${ }^{2}$, Mitsuru Akashi ${ }^{1, *}$ \\ ${ }^{1}$ Department of Applied Chemistry, Graduate School of Engineering, Osaka University, \\ 2-1 Yamadaoka, Suita, Osaka 565-0871, Japan \\ ${ }^{2}$ Bristol Musculoskeletal Research Unit, Clinical Science at North Bristol University of Bristol, \\ Avon Orthopaedic Centre, Southmead Hospital, Bristol BS10 5NB, United Kingdom
}

\section{CORESPONDING AUTHOR FOOTNOTE}

*Author to whom correspondence should be addressed:

Professor Mitsuru Akashi

Tel +81-6-6879-7356

Fax $+81-6-6879-7359$

E-mail: akashi@chem.eng.osaka-u.ac.jp 


\begin{abstract}
Three-dimensional (3D) tissue constructs consisting of human cells have opened a new avenue for tissue engineering, pharmaceutical and pathophysiological applications, and have great potential to estimate the dynamic pharmacological effects of drug candidates, metastasis processes of cancer cells, and toxicity expression of nano-materials, as a 3D-human tissue model instead of in vivo animal experiments. However, most 3D-cellular constructs are a cell spheroid, which is a heterogeneous aggregation, and thus the reconstruction of the delicate and precise 3D-location of multiple types of cells is almost impossible.

In recent years, various novel technologies to develop complex 3D-human tissues including blood and lymph capillary networks have demonstrated that physiological human tissue responses can be replicated in the nano/micro-meter ranges. Here, we provide a brief overview on current 3D-tissue fabrication technologies and their biomedical applications. 3D-human tissue models will be a powerful technique for pathophysiological applications.
\end{abstract}

Keywords: 3D constructs; human-tissue model; layer-by-layer assembly; vasculature; extracellular matrix; nano-films

\footnotetext{
Abbreviations:

3D, three-dimension; LbL, layer-by-layer; ECM, extracellular matrix; FN, fibronectin: G, gelatin; NHDF, normal human dermal fibroblasts; HUVEC, human umbilical vein endothelial cells; iPS, induced-pluripotent stem
} 


\section{Introduction}

Quantitative and time-lapse analyses in microenvironments for drug diffusion, drug toxicity, and the release of molecules from drug delivery carriers are useful to explore the physiological processes, mechanisms, and treatments of disease or injury through pathophysiology. Various spatial analytical instruments such as computed tomography, magnetic resonance imaging, and positron emission tomography have been employed for general in vivo animal experiments in pathophysiology. However, even though their detection limits have been improved day by day, the detection of events in the micron-meter scale is still difficult. Moreover, species differences between animals and humans can cause fundamental confounders such as metabolic processes, enzymes, and membrane proteins. Accordingly, in vitro human cell-based drug evaluations, including drug efficacy testing, toxicology, and basic cell biology [1,2], are of great importance as an alternative to animal experiments to solve the significant issue of species differences. In particular, animal testing for cosmetics and chemicals has been prohibited under the $7^{\text {th }}$ Amendment to the Cosmetics Directive (Council Directive 76/768/EEC) and REACH (registration, evaluation, authorization and restriction of chemicals) in the European Union (EU) from March $2013[3]$.

Cell-based drug evaluations have generally been performed in a plastic dish under monolayer (two-dimensional; 2D) cell conditions. Since nearly all tissues are integrated three-dimensional (3D) structures of multiple types of cells and extracellular matrices (ECMs), and since intercellular signaling is important for biological functions, it is generally difficult to estimate the actual drug effects on physiologic functions by $2 \mathrm{D}$-culture methods. The development of 3D human tissue constructs consisting of simplified tissue structures with multiple types of cells and ECMs is a key challenge for pharmaceutical and pathophysiological evaluations and tissue engineering.

In general, a cell spheroid has been employed as the 3D-culture model, especially 3D-cancer spheroids for metastasis or pharmacological assays of cancer cells [4-6] and 3D-hepatocyte 
spheroids for inducing higher activity of hepatocytes [7-9]. Although, interesting 3D-co-culture spheroids have been reported to investigate tumor invasion [10,11], to enhance hepatocyte functions [12] and to mimic in vivo conditions [13], the reconstruction of the delicate and precise 3D-location of multiple types of cells has not been achieved yet due to their heterogeneity, the lack of control of the cell number and location, and necrosis inside the cells because of insufficient nutrients.

Here we provide a brief overview of the technologies for the construction of 3D-tissue constructs, mainly our hierarchical cell manipulation technologies, and then discuss how these 3Dtissues could contribute to understanding nano-pathophysiology.

\section{Top-down approach for development of 3D-tissue constructs}

There are basically two kinds of approaches, top-down and bottom-up approaches, for the construction of 3D-tissue constructs (Figure 1). A top-down approach has been reported historically, especially the biodegradable scaffold method. Biodegradable scaffolds and hydrogels consisting of biodegradable polymers, such as poly(lactic acid), poly(glycolic acid), alginate and collagens have been used for the construction of 3D-constructs containing living cells $[14,15]$. The topological control of biodegradable porous scaffolds [16], especially nanofiber scaffolds by electrospinning [17] or self-assembling amphiphilic peptides [18], has attracted much attention due to their high porosity and the controlled alignment of the fibers to control cellular function. The cells encapsulated in the scaffolds can growth actively, and finally formed random cell aggregations inside it. Although their growth rate can be controlled using growth factors in the culture medium, 3D-engineered tissues possessing precisely-controlled cell types, cell alignment, and cell-cells interaction have not been developed yet. These nanofiber scaffolds can contribute to the tentative cell alignment or adhesion due to morphology, but it is difficult to maintain these effects, because the nanofibers are covered completely with cultured cells and expressed ECMs from the cells. Accordingly, a conventional approach using biodegradable matrices such as 
hydrogels or fiber scaffolds seems to have several limitations in developing 3D-tissue constructs which satisfy the above requirements. A bottom-up approach using multiple cell types as pieces of tissue has recently attracted much attention to solve these problems.

\section{Bottom-up approach for the development of 3D-tissues}

Various bottom-up approaches such as cell sheets [19], magnetic liposomes [20], hierarchical cell manipulation [21], polymeric aqueous two-phase systems [22] and printing cells and polymers [23] have been reported in constructing a complex tissue structure. These bottom-up approaches are generally categorized into two groups: cell-based methods, and cell and polymer-based methods. Here, the characteristics, advantages, and issues with both approaches are briefly summarized.

\subsection{Cellular layer-by-layer approaches}

One of the most general methods of the bottom-up approaches is cellular layer-by-layer (LbL). Okano and co-workers have reported the fabrication of monolayers of cell sheets and their stacking to create multilayered structures using temperature-responsive polymer grafted culture dishes [19]. Using temperature-responsive dishes, cultured cells can be harvested as fragile sheets by temperature changes, thereby avoiding the use of proteolytic enzymes. They prepared multiple sheet structures of cardiomyocytes for the reconstruction of 3D-myocardical tissues by stacking the cell sheets [24]. Because the cell sheets have ECM at the bottom underneath the sheet, they can stack easily due to the interactions with cell membrane receptors. This is one of the leading methods to fabricate multilayered cellular structures.

Although the cell sheet is intriguing method, the use of the fragile sheets is not easy for layman. The other method to bind cell to form 3D-structures, magnetic force has been reported from Ito and co-workers [20]. They applied a magnetic force to construct a heterotypic, layered co-culture system of hepatocytes and endothelial cells that was not limited by the cell type. Magnetic cationic 
liposomes with positively-charged surfaces were adsorbed onto the cell surfaces, and then magnetic forces were applied to create multilayered structures. This method can fabricate multilayered structures of cells, independent of the cell type, because the driving force is magnetic. Although the remnant amount of magnetic liposomes is very small, they still might induce toxicity.

Takayama and co-workers reported a new approach, a biphasic system for non-contact cell printing on cells [22]. Aqueous solutions of polyethylene glycol (PEG) and Dextran (Dex) above a certain concentration, and segregate and form a two-phase system with Dex always forming the bottom phase. They controlled the interfacial tension of each phase to achieve cell printing on cells, and reported that cellular niches supported the neuronal differentiation of mouse embryonic stem cells (mESC) and showed that the density of printed mESC is an important factor for guiding mESC differentiation into neurons. This is a unique technique to prepare bilayered cellular constructs, and thus it is expected to be applicable for over three layers.

\subsection{Nano-ECM assisted Cellular LbL}

To develop a simple, bottom-up approach, we focused on the cell adhesive properties of the ECM surrounding the cell microenvironment. In most in vitro cell culture methods, researchers obtain only cellular monolayers, probably due to the absence of sufficient ECM on the cell surfaces. If cell adhesive nanometer-sized materials like an ECM were fabricated directly on cell membrane surfaces, then the 3D-control of cell-cell adhesion would be possible. We focused on the LbL technique, which is an appropriate method to prepare nano-meter sized films on a substrate by alternate immersion into interactive polymer solutions [25,26]. The appropriate choice of natural ECM components for the preparation of nanofilms is important to avoid cytotoxicity, and the typical ECM presents with cell-adhesive moieties such as RGD (arginine-glycine-aspartic acid) and other functional moieties [27]. We selected fibronectin $(\mathrm{FN})$ and gelatin $(\mathrm{G})$ to prepare nanoECM films on the cell surface. $\mathrm{FN}$ is a flexible multifunctional glycoprotein, and plays an important role in cell attachment, migration, differentiation, and so on [28,29]. FN is well-known 
to interact not only with a variety of ECM proteins such as collagens (gelatins) and glycosaminoglycans, but also with the $\alpha_{5} \beta_{1}$ integrin receptor on the cell surface [30]. We have previously reported FN-based LbL multilayers composed of FN and ECM components, such as gelatin, heparin, and elastin, and was constructed by alternate immersion into the solutions (LbL assembly) [31]. Although FN and G have a negative charge under physiological conditions, they interact with each other because FN has a collagen binding domain [29], indicating the different driving forces of polyelectrolyte (PE) films using polycations. Thus, the FN-G nanofilms were expected to provide a suitable cell-adhesive surface similar to the natural ECM for the second layer of cells, without any cytotoxicity. That is the key idea behind the method for cell LbL, "hierarchical cell manipulation" (Figure 2) [21,32-34].

The fabrication of 3D-cellular multilayers composed of cells and FN-G nano-ECM films was performed according to the following process [21]. Briefly, the LbL assembly of FN and G onto the cell surface was analyzed quantitatively using a quartz crystal microbalance (QCM) as the assembly substrate, and with a phospholipid bilayer membrane. The mean thickness of the LbL assembly after 1, 7, and 23-steps was calculated to be 2.3, 6.2, and $21.1 \mathrm{~nm}$, respectively. The top and cross sections of the confocal laser scanning microscopic (CLSM) 3D-merged images indicated a homogeneous assembly of fluorescently-labeled FN-G nanofilms on the surface of mouse L929 fibroblasts. We fabricated a bilayer of mouse L929 fibroblast cells with or without FN-G nanofilms using a cover glass as a substrate. When the 7-step assembled FN-G nanofilms were prepared on the surface of the first L929 cell layer, the second layer cells were then observed on the first cell layer. However, when the nanofilm was not prepared or the 1-step-assembled nanofilm (only FN) was assembled on the first cell layer, then the bilayer architecture was not observed. These results suggested that a $2.3 \mathrm{~nm}$ thick FN film was inadequate, and at least approximately $6 \mathrm{~nm}$ of $\mathrm{FN}-\mathrm{G}$ nanofilm was required as a stable adhesive surface for the second cell layer. Four layered (4L) cellular multilayers were clearly observed after four repetitions of these steps by CLSM and hematoxylin and eosin (HE) staining. The thickness of the obtained 
multilayers estimated from the 3D reconstructed CLSM images increased linearly with increasing cell layer number. This hierarchical cell manipulation technique could be applied to various types of cells, not only the fibroblast cells, but also myoblasts, cardiac myocytes, smooth muscle cells (SMC), hepatocytes, and tumor fibrotic fibroblasts (Figure 3) [35]. Furthermore, using a combination of multiple types of cells, e.g. SMC and endothelial cells and fibroblasts and keratinocytes, it was possible to fabricate human blood vessels and skin models, respectively [33].

\subsubsection{D-Cell assembling approaches}

Cellular LbL methods are useful to construct multilayered structures one by one, but it takes a long time to fabricate thick tissue constructs because an almost half day incubation is necessary for stable cell adhesion on the cell surfaces. Thus, 3D-cell assembling approaches have recently attracted much attention. Takeuchi and Matsunaga reported a molding technique of cell beads, which are collagen gel beads covered with adhered cells [36]. The cell beads are molded into a custom silicone chamber to form the macroscopic 3D-tissue constructs. In the mold, the cell beads can adhere to each other via the cells coated onto the collagen gel beads. Finally, since the collagen gel beads were degraded by enzymes secreted from the cells, the molded tissues can be released. They reported improvements in the amount of albumin secreted from HepG2 cells by co-culture with fibroblast cells in the constructed 3D-tissues. The construction of complex macroscopic 3D tissues using multiple cell types is expected using this method.

We recently developed a simple and rapid bottom-up approach, called the cell-accumulation technique, by single cell coating using FN-G nanofilms [34]. Since the FN-G nanofilms prepared on individual cell surfaces provide an interactive property with the $\alpha_{5} \beta_{1}$ integrin receptor of the cell membrane, the cell-cell adhesion of all seeded cells in three-dimensions can be induced at the same time (Figure 4, the "cell accumulation technique").

The fabrication of approximately $6 \mathrm{~nm}$ thick FN-G films onto a dispersed single cell surface was performed based on our previous reports for the preparation of PE LbL nanofilms onto silica 
particles [37]. The cell viability after preparing the FN-G nanofilms was evaluated, and compared to the PE films. In the case of the FN-G nanofilm, almost complete survival was confirmed (>98\%), whereas the other nanofilms prepared by electrostatic driving forces showed cytotoxicity dependent on their LbL step number. We also confirmed over 91\% cell viability for NHDFs, HUVECs, and human hepatocellular carcinoma (HepG2) cells after preparing these FN-G films. After coating with the FN-G nanofilms, $2 \times 10^{6}$ NHDFs were incubated in a cell culture insert for one day. 8L-dense constructs of $35 \pm 4 \mu \mathrm{m}$ thicknesses were successfully obtained, whereas a tattered and porous structure was barely obtained using non-coated cells. The thickness of the obtained tissue constructs increased upon increasing the volume of the culture medium, and thus the current maximum thickness is over $100 \mu \mathrm{m}$ for greater than 20L-structures. More importantly, the construction of approximately 20L-structures per one day after only a nanofilm coating within 1 hour has never been reported previously, and this approach has tremendous versatility for various cells.

\subsubsection{Higher cellular functions in 3D-tissue constructs than in 2D monolayers}

Cellular functions in the 3D-tissue constructs are expected to be higher than those of monolayered cells in general culture. Some researchers have reported the functions of layered cellular architectures in vitro [38]. However, the basic properties of 3D-cellular structures, such as their layer number or the cell types, have not been clarified yet.

We evaluated the structural stability of layered constructs consisting of normal human dermal fibroblasts (NHDFs) and human umbilical vein endothelial cells (HUVECs) in relation to their layer number [39]. To evaluate the general effects of 3D-layered structures on cellular stress or inflammation, we purposely fabricated biologically-meaningless layered structures consisting of endothelial cells and fibroblasts. Dulbecco's modified Eagle's medium (DMEM) containing 10\% feral bovine serum (FBS), a general culture medium, was employed in this study to avoid the influence of specific growth factors. Interestingly, the HUVECs adhered homogeneously onto the 
surface of 4L-NHDFs, and tight-junction formation was widely observed at the centimeter scale, whereas heterogeneous HUVEC domain structures were observed on the monolayered (1L) NHDFs. The production of heat shock protein 70 (Hsp70) and IL-6 from the cellular structures was investigated to elucidate any 3D-structural effect on cellular function. The Hsp70 expression of the HUVECs decreased after adhesion onto the 4L-NHDF structure as compared with a HUVEC monolayer. Surprisingly, the Hsp70 production response to heat shock increased drastically by approximately 10-fold as compared with a non-heat shock after 3D structure formation, whereas the monolayer structures showed no change. Moreover, the production of the inflammatory cytokine IL-6 decreased significantly depending on the layer number of NHDFs. These results suggest that the 4L-NHDF provided a more favorable environment for the HUVECs than a cell culture plastic disk to induce high thermosensitivity, and to suppress the inflammatory response from the substrate. Since the FN-G nanofilms prepared on the cell surface did not affect cellular functions [40], we concluded that the layered construct would be an analogous environment to natural tissues. These findings could be important for not only tissue engineering, but also for basic cell biology.

\subsubsection{Potential application of 3D-tissue constructs for pharmaceutical assays}

Constructed 3D-tissue structures are desirable as a human tissue model for pathophysiological assays. However, since a 3D-human tissue models have not been reported much, their application reports are also few. We tried to demonstrate the potential in vitro applications of 3D-tissue constructs as a human tissue model for pharmaceutical assays.

3D-blood vessel models consisting of human SMCs and HUVECs were fabricated by hierarchical cell manipulation [41]. The 5L-blood vessel models consisting of 4L-umbilical artery SMC (UASMC) and an outermost 1L-HUVEC showed histological staining patterns similar to that of human blood vessels, and the surface of the 4L-UASMC/1L-HUVEC revealed high biocompatibility that avoided the adsorption and activation of platelets in human platelet-rich 
plasma (PRP). We tried to use 5L-artery models consisting of human aortic SMCs and endothelial cells (ECs) to analyze the production and diffusion of nitric oxide (NO) from ECs in response to the peptide hormone bradykinin [42]. The nitric oxide (NO) produced from the ECs diffuses into the SMCs through their cell membranes, and activates guanylate cyclase to produce intracellular cyclic guanosine monophosphate (cGMP), which induces a signaling pathway mediated by kinase proteins leading to SMC relaxation [43]. Accordingly, quantitative, kinetic, and spatial analyses of the extracellular delivery of NO molecules from the EC layer to the SMC layers upon drug stimulation are crucial for the pharmaceutical and biomedical evaluations of hypertension and diabetes. Recently, a significant correlation between NO production and diabetes mellitus was clarified, e.g. reduced NO production in both type 1 and 2 diabetes [44], and also increases in NO production related to improvements in type 2 diabetes were reported [45]. Thus far, pharmaceutical assays of NO production have been performed by in vivo animal experiments, but low reproducibility and differences in NO production depending on the animal types remain unsolved issues. Thus, the development of a convenient and versatile method for in vitro quantitative and spatial analyses of NO diffusion inside the artery wall instead of animal experiments is important for biological and pharmaceutical applications.

Briefly, we demonstrate for the first time spatial and quantitative analyses of NO diffusion from the EC layer to the SMC layers using a 3D artery model with sensor particles [37] allocated into each cellular layer. The 3D structural effect of ECs and SMCs on NO procution from the ECs was clarified in relation to the direction of interaction between these cells. Furthermore, a graded concentration change of NO from the uppermost EC layer to the underlying SMC layers was elucidated by 3D analysis using CLSM. This method does not require special instruments or techniques and is convenient and effective for drug screening, and thus it has possibility to be a solution of general animal experiments.

\subsection{Cell and polymer-based approaches}


These cell-based approaches allow us to build a 3D-tissue construct with high cell density which reveals cell-cell interactions. However, the obtained constructs are still far from the real in vivo tissues. In particular, the mechanical properties and size of the obtained tissues are significantly lower and smaller, respectively, than those of real tissues because of the ECM components. Complex ECM fiber networks play an important role for stiffness and elasticity of our tissues and organs. The development of composite tissues consisting of not only cells, but also polymers or ECM proteins will be important for the construction of 3D-tissue analogs with similar mechanical and morphological properties.

To control the cellular location in the matrix, inkjet printing of cells and polymers has been employed [23]. Mironov and co-workers demonstrated the 3D-construction of complex structures by printing in situ crosslinkable polymers and ECM solutions containing cells [46,47]. Nakamura et al. reported the ability to write lines about $50 \mu \mathrm{m}$ wide by printing cells suspended in sodium alginate onto a thin film of calcium chloride due to the formation of alginate gels through coordinate bonding with calcium ions [48]. Both methods reported the fabrication of free-standing tubular constructs containing living cells, and their mechanical and morphological properties were clearly higher than those of the products from cell-based approaches. However, the encapsulated cells were isolated inside the matrix, and no physiologic functions as a living tissue were observed until their death after a couple of days of incubations. Furthermore, the obtained 3D-constructs were not stable for long-term cultures (more than 1 week) due to enzymatic degradation or hydrolysis. The variety of in situ crosslinkable polymers is also limited. The challenge here is in developing new biomaterials to allow high cell adhesion, cell-cell interactions, and long-term stability.

\section{Development of vascularized 3D-tissue constructs}

Vascularization in 3D-tissue constructs is still a key challenge in the tissue engineering field to prevent cell necrosis inside thick 3D-tissue constructs. Since the phenomenon of in vitro 
angiogenesis was discovered by Folkman and co-authors in 1979 [49,50], researchers have tried to control the capillary formation of endothelial cells in scaffolds [51] and reconstructed 3D-tissues [52,53]. However, it is difficult to control the alignment of the reconstructed blood capillary. Recently, micro-fluidic systems [54] and channel preparation in hydrogels [55] have been reported to construct precisely-controlled capillary networks by covering the inner-surface of pores with endothelial cells. Since the morphology and biological properties of the endothelialized microchannels resembled in vivo blood capillaries, these technologies are expected to be useful for the in vitro study of angiogenesis. Currently, the development of perfusable blood capillary networks has been reported by connecting ex vivo tissue specimens to act as a vascular bed [56]. Because of the high performance to create blood flow inside these capillary networks, their applications for biomedical and pathophysiology fields are expected.

\subsection{Scaffold-based approaches}

Unger et al. reported the formation of microcapillary-like structures of human dermal microvascular endothelial cells on 3D-porous bone biomaterials such as hydroxyapatite or calcium phosphate by co-culture with osteoblast cells or an osteoblast-like cell line [51]. These microcapillary-like structures were interwined between the cell layers of the osteoblasts, and did not form when exogenous angiogenic stimuli were added to these co-cultures. The co-culture model presented the experimental possibility to delineate how specific genes are regulated as a result of cell-cell interactions involving endothelial cells and osteoblasts. Langer et al. reported the induction of endothelial vessel networks in engineered skeletal muscle tissue constructs using a 3D multi-culture system consisting of myoblasts, embryonic fibroblasts and endothelial cells co-seeded on highly porous, biodegradable polymer scaffolds [52]. They found that the addition of embryonic fibroblasts increased the levels of vascular endothelial growth factor expression in the construct, and promoted the formation and stabilization of the endothelial vessels. Using in vivo 
animal models, improvements in the vascularization, blood perfusion and survival of the muscle tissue constructs after implantation was clearly confirmed.

These are leading reports to demonstrate in vitro vascularization in 3D-scaffolds, and the latter clearly support the benefit of pre-vascularization in the scaffold for in vivo disease treatments. However, the construction of in vitro perfusable vascular networks is still a key challenge. Chen and co-workers reported perfusable network formation by a sacrificial template approach [55]. They printed rigid 3D filament networks of carbohydrate glass, and used them as a cytocompatible sacrificial template in engineered tissues containing living cells to generate cylindrical networks that could be lined with endothelial cells and perfused with blood under high-pressure pulsatile flow. Since this simple vascular casting approach allows independent control of the network geometry, endothelialization and the extravascular tissues, it is compatible with a wide variety of cell types, synthetic and natural extracellular matrices, and crosslinking strategies. They also demonstrated that the perfused vascular channels sustained the metabolic function of primary rat hepatocytes in engineered tissue constructs.

\subsection{Microfluidic approaches}

The pharmaceutical assay system in in vitro vascularized 3D-tissue chips will be suitable for applications in pathophysiological research. Zheng et al. developed living microvascular networks in 3D-tissue scaffolds using a microfluidic system, and demonstrated their biofunctionality in vitro [54]. They used the lithographic technique to form endothelialized microfluidic vessels within a native collagen matrix, and elucidated the angiogenic activities of the endothelium and differential interactions with prevascular cells seeded in the collagen bulk. Moreover, the non-thrombotic nature of the vascular endothelium and its transition to a prothrombotic state during an inflammatory response were demonstrated. This microfluidic system shows broad potential for the study of cardiovascular biology and pathophysiology. 
Jeon and co-workers reported a microfluidic-based platform whereby they model natural cellular programs found during normal development and angiogenesis to form perfusable networks of intact 3D microvessels, as well as tumor vasculatures, based on the spatially-controlled coculture of endothelial cells with stromal fibroblasts, pericytes and cancer cells [57]. Chen et al. also discovered a biomimetic model to reconstitute angiogenic sprouting morphogenesis [58]. They reported an in vitro 3D biomimetic model that reconstitutes the potential utility of the model to elucidate the molecular mechanisms that coordinate the complex series of events involved in neovascularization.

\subsection{Sandwich co-culture approaches}

The microfluidic approaches and sacrificial-template methods have recently attracted attention for designed microvascular networks, because their morphology and structure are easily controlled. However, the long-term stability of the obtained microcapillary constructs is still issue because matrices such as collagen gels are easily decomposed by enzymes expressed from the cells. This instability is closely related to the number of seeded cells, because the amount of expressed enzymes is dependent on the cell number. Furthermore, since the number of surrounding cells is quite low and they do not contact the microvascular networks, it is difficult to evaluate actual drug or cell permeations from the microvascular networks into the surrounding cells. To develop actual vascularized 3D-tissue constructs, the surrounding cells should interact strongly with the microvascular endothelial cells. The embedded structures of the micro-vasculatures in the 3Dcellular tissues will be the morphology of real in vivo tissues.

Okano et al. reported the development of 3D-microcapillaries embedded into stacked cell sheets [53]. They also observed morphological changes of the HUVECs into network structures when the cells were sandwiched between two myoblast sheets. Moreover, the endothelial networks in the five-layer myoblast sheet constructs were observed to connect to the host vessels after transplantation into the subcutaneous tissues of nude rats, resulting in a neovascularization that 
allowed the graft to survive. These prevascularized 3D-tissues possessed the obvious embedded structures of the microvasculatures in the cell sheet tissues, which might reflect the actual relationship between a blood capillary and the surrounding cells. Currently, the development of perfusable blood capillary networks has been reported by connecting ex vivo tissue specimens to act as a vascular bed [56]. Because of the high performance to create blood flow inside the capillary networks, their applications for the biomedical and pathophysiology fields are expected. For pathophysiological applications, the construction of vascularized 3D-tissue arrays in general multi-well plates will be a powerful tool.

We demonstrated the fabrication of vascularized 3D-tissue arrays, which were constructed in 12 and 24 micro-well plates using the cell accumulation technique [34]. The construction of thick multilayered tissues with endothelial tube networks by embedding HUVECs in 3D-tissues composed of NHDFs has been achieved by sandwich culture. After 2 7 days of incubation, highly-developed capillary networks and a tubular morphology of the HUVECs were clearly observed by CLSM (Figure 5). A dense and homogeneous vascularized network in the multilayered tissues of $1 \mathrm{~cm}$ width and $50 \mu \mathrm{m}$ height was confirmed in 12 and 24 micro-well cell culture inserts. The percentage occupied area and distance of this capillary network of HUVECs was calculated to be approximately $63 \pm 12 \%$ and $50 \sim 150 \mu \mathrm{m}$ by image analysis, respectively. This would provide a benefit for the surrounding cells, because cells in living tissues require endothelial tube networks within a perimeter of 100-150 $\mu \mathrm{m}$ to supply oxygen. Moreover, lymph capillary network structures could also be obtained by employing lymph endothelial cells instead of HUVECs. When we used both blood and lymph endothelial cells for the vascularization process, individual networks in 3D-NHDF tissue constructs were successfully obtained. The obtained vascularized constructs were stable during long-term culture for at least 3 weeks. These microvascular and -lymph networks in multi-well plates will be powerful tools for both pharmaceutical and pathophysiological applications. 


\section{Future directions}

Various unique technologies for the construction of 3D-human complex tissues have been discovered for basic research in pathophysiology and tissue engineering. Since multiple types of human cells are assembled together to build up tissue architectures, the theory for 3D-tissue constructs will be established sooner. The future will move to the next step, the reconstruction of "functional" 3D-tissue constructs. Herein, the term "function" includes issues such as the longterm stability of the 3D-structures as a tissue, functional properties secondary to containing functional cells or tissues, and high repeatability of the structures and functions for commercialization.

Regarding long-term stability, we currently reported one of the most important functions as a tissue, the structural stability of the 3D-constructs [59]. In this paper, we demonstrated that 5LNHDF tissues maintained their original thickness, living-cell density and layer number even after 1 month of culture. Indeed, apoptotic cells were found in the tissues during cell culture, but the living cells seemed to proliferate to fill up the space which was generated by the detachment of the dead cells. Although longer-term evaluations have not been performed yet, the maintenance of 3Dcomplex architectures will be important for their applications related to vascularization.

More practical functions need to be proved in a 3D-human tissue model. For example, an important function of the blood capillary is the permeability of molecules or cells. Jeon et al recently reported the fabrication of perfusable 3D-microvascular networks on a microfluidic chip [57]. We also demonstrated the development of multilayered blood capillaries in biodegradable hydrogels, and the analysis of the differential permeability of albumin molecules inside the capillaries as compared to the tube without cells (Figure 6) [60]. Multilayered blood vessel structures revealed an inhibition of the leakage of albumin molecules, whereas the tube structures without the surface coverage by cells showed high leakage of the molecules. For the other example, 3D-human placental models constructed by the cell accumulation technique indicated for the first time a sensitive response to the environmental changes of oxygen partial pressure, the same as the 
in vivo human placenta [61]. Briefly, we previously reported nanoparticle toxicity using 2Dtrophoblast barriers prepared by employing the BeWo human cell line $[62,63]$, but the responses and functions of these barriers were poor as compared to actual trophoblast barriers, due to the 2D structures and cell lines. When 3D-trophoblast models were developed using human primary trophoblasts by this technique, the models revealed almost the same response to the change in environmental factors. This is a good example to provide the biological and biochemical evidence for reconstructed 3D-cell constructs as a human functional tissue model for pathophysiological applications.

Regarding the industrial production of 3D-tissue constructs, automation of the fabrication process is important for high repeatability of the structures and functions. Moreover, automation is also effective to prevent any contamination caused by manual procedures. As described above, the automatic fabrication of 3D-tissue constructs, e.g. ink-jet printing and prototyping technologies, has recently attracted much attention (Figure 6) $[23,64]$. The precise control of the cell and polymer locations by printing or dispensing can allow us to construct complex 3D structures which reflect the structures of natural tissues or organs. Such "biofabrication" techniques are starting to play an important role in this field. The combination between biofabrication and human inducedpluripotent stem (iPS) cells [65] is expected to open new avenues for research in pathophysiology because iPS cells have great potential for high replication and differentiation. They also have the potential to provide an unlimited source of cells for a variety of applications, particularly in vitro tailor-made drug screening [66]. Furthermore, since disease-specific iPS cells such as Alzheimer disease or Parkinson's disease will be obtained, the development of disease-specific 3D-tissues might be feasible in the future, which is important for drug discovery and mechanism solution. Normal and diseased 3D-tissue constructs fabricated in a general micro-well plate will be applicable alternative for screening assays and pathophysiology. 


\section{Conclusions}

Here, we described an overview on current 3D-tissue fabrication technologies and their biomedical applications, including our two techniques, the hierarchical cell manipulation and the cell accumulation technique. 3D-tissue constructs consisting of human cells have opened a new avenue as a key tool for tissue engineering, and pharmaceutical and pathophysiological applications. Regarding iPS cell technologies, the tissue engineering approach for the construction of 3D-human tissue constructs must be investigated in the near future. 


\section{Acknowledgements}

This work was supported mainly by PRESTO-JST, and partly by an Industrial Technology Research Grant Program in 2006 (06B44017a) from NEDO of Japan, a Grant-in-Aid for Scientific

Research on Innovative Areas (21106514) from MEXT of Japan, The Noguchi Institute Fund, and by NEXT Program from JSPS (LR026). 


\section{References}

[1] J. Drewe, X. Cai, Cell-based apoptosis assays in oncology drug discovery, Expert. Opin. Drug Discov. 5, (2010) 583-596.

[2] E. Michelini, L. Cevenini, L. Mezzanotte, A. Coppa, A. Roda, Cell-based assays: fuelling drug discovery, Anal. Bioanal. Chem. 398 (2010) 227-238.

[3] Commission staff working documents, Time tables for the phasing-out of animal testing in the framework of the $7^{\text {th }}$ amendment to the cosmetics directive (council directive 76/768/EEC; EN, SEC82004) 1210 (2004).

[4] J. Debnath, J. S. Brugge, Modelling glandular epithelial cancers in three-dimensional cultures, Nat. Rev. Cancer 5, (2005) 675-688.

[5] G. Y. Lee, P. A. Kenny, E. H. Lee, M. J. Bissell, Three-dimensional culture models of normal and malignant breast epithelial cells, Nat. Methods 4, (2007) 359-365.

[6] H. Dolznig, A. Walzl, N. Kramer, M. Rosner, P. G.-Chesa, M. Hengstschläger, Organotypic spheroid cultures to study cumor-stroma interaction during cancer development, Drug Discov. Today: Dise. Mod. 8, (2011) 113-119.

[7] N. Koide, T. Shinji, T. Tanabe, K. Asano, M. Kawaguchi, K. Sakaguchi, Y. Koide, M. Mori, T. Tsuji, Continued high albumin production by multicellular spheroids of adult rat hepatocytes formed in the presence of liver-derived proteoglycans, Biochem. Biophys. Res. Commun. 161, (1989) 385-391.

[8] H. Otsuka, A. Hirano, Y. Nagasaki, T. Okano, Y. Horiike, K. Kataoka, Two-dimensional multiarray formation of hepatocyte spheroids on a microfabricated PEG-brush surface, ChemBioChem 5, (2004) 850-855.

[9] J. Fukuda, Y. Sakai, K. Nakazawa, Novel hepatocyte culture system developed using microfabrication and collagen/polyethylene glycol microcontact printing, Biomaterials 27, (2006) 1061-1070. 
[10] L. de Ridder, M. Cornelissen, D. de Ridder, Autologous spheroid culture: a screening tool for human brain tumour invasion, Crit. Rev. Oncol. Hematol. 36, (2000) 107-122.

[11] T. Brabletz, A. Jung, S. Reu, M. Porzner, F. Hlubek, L. A. K.-Schughart, R. Knuechel, T. Kirchner, Proc. Natl. Acad. Sci. USA 98, (2001) 10356-10361.

[12] H.-F. Lu, K.-N. Chua, P.-C. Zhang, W.-S. Lim, S. Ramakrishna, K. W. Leong, H.-O. Mao, Three-dimensional co-culture of rat hepatocyte spheroids and NIH/3T3 fibroblasts enhances hepatocyte functional maintenance, Acta Biomater. 1, (2005) 399-410.

[13] T. T. Goodman, C. P. Ng, S. H. Pun, 3-D tissue culture systems for the evaluation and optimization of nanoparticle-based drug carriers, Bioconjugate Chem. 19, (2008) 1951-1959.

[14] R. Langer, J. P. Vacanti, Tissue engineering, Science 260, (1993) 920-926.

[15] K. Y. Lee, D. J. Mooney, Hydrogels for tissue engineering, Chem. Rev. 101, (2001) 18691879.

[16] J. Lee, M. J. Cuddihy, N. A. Kotov, Three-dimensional cell culture matrices: state of the art, Tissue Eng.: Part B 14, (2008) 61-86.

[17] Y. Dzenis, Spinning continuous fibers for nanotechnology, Science 304, (2004) 1917-1919.

[18] S. Zhang, Fabrication of novel biomaterials through molecular self-assembly, Nat. Biotechnol. 21, (2003) 1171-1178.

[19] J. Yang, M. Yamato, C. Kohno, A. Nishimoto, H. Sekine, F. Fukai, T. Okano, Cell sheet engineering: Recreating tissues without biodegradable scaffolds, Biomaterials 26 (2005), 6415-6422.

[20] H. Akiyama, A. Ito, Y. Kawabe, M. Kamihira, Genetically engineered angiogenic cell sheets using magnetic force-based gene delivery and tissue fabrication techniques, Biomaterials 31 , (2010) 1251-1259.

[21] M. Matsusaki, K. Kadowaki, Y. Nakahara, M. Akashi, Fabrication of cellular multilayers with nanometer-sized extracellular matrix films, Angew. Chem. Int. Ed. 46, (2007) 4689-4692. 
[22] H. Tavana, B. Mosadegh, S. Takayama, Polymeric aqueous biphasic systems for non-contact cell printing on cells: Engineering heterocellular embryonic stem cell niches, Adv. Mater. 22, (2010) 2628-2631.

[23] B. Derby, Printing and prototyping of tissues and scaffolds, Science 338, (2012) 921-926.

[24] T. Shimizu, M. Yamato, Y. Isoi, T. Akutsu, T. Setomaru, K. Abe, A. Kikuchi, M. Umezu, T. Okano, Fabrication of pulsatile cardiac tissue grafts using a novel 3-dimensional cell sheet manipulation technique and temperature-responsive cell culture surfaces, Circ. Res. 90, (2002) e40-e48.

[25] G. Decher, J. D. Hong, Buildup of ultrathin multilayer films by a self-assembly process, 1: Consecutive adsorption of anionic and cationic bipolar amphiphiles on charged surfaces, Makromol. Chem. Macromol. Symp. 46, (1991) 321-327.

[26] G. Decher, Fuzzy nanoassemblies: Toward layered polymeric multicomposites, Science 277, (1997) 1232-1237.

[27] H. K. Kleinman, D. Phlip, M. P. Hoffman, Role of the extracellular matrix in morphogenesis, Curr. Opin. Biotechnol. 14, (2003) 526-532.

[28] K. M. Yamada, Cell surface interactions with extracellular materials, Ann. Rev. Biochem. 52, (1983) 761-799..

[29] R. O. Hynes, Fibronectins. New York: Springer-Verlag Inc. 1990.

[30] E. Ruoslahti, M. D. Pierschbacher, New perspectives in cell adhesion: RGD and integrins, Science 238, (1987) 491-497.

[31] Y. Nakahara, M. Matsusaki, M. Akashi, Fabrication and enzymatic degradation of fibronectinbased ultrathin films, J. Biomater. Sci. Polymer Edn. 18, (2007) 1565-1573.

[32] M. Matsusaki, H. Ajiro, T. Kida, T. Serizawa, M. Akashi, Layer-by-layer assembly through weak interactions and their biomedical applications, Adv. Mater. 24 (2012) 454-474.

[33] M. Matsusaki, Development of three-dimensional tissue models based on hierarchical cell manipulation using nanofilms, Bull. Chem. Soc. Jpn. 85, (2012) 401-414. 
[34] A. Nishiguchi, H. Yoshida, M. Matsusaki, M. Akashi, Rapid construction of threedimensional multilayered tissues with endothelial tube networks by the cell-accumulation technique, Adv. Mater. 23, (2011) 3506-3510.

[35] H, Hosoya, K. Kadowaki, M. Matsusaki, H. Cabral, H. Nishihara, H. Ijichi, K. Koike, K. Kataoka, K. Miyazono, M. Akashi, M. R. Kano, Engineering fibrotic tissue in pancreatic cancer: A novel three-dimensional model to investigate nanoparticle delivery, Biochem. Biophys. Res. Commun. 419, (2012) 32-37.

[36] Y. T. Matsunaga, Y. Morimoto, S. Takeuchi, Molding cell beads for rapid construction of macroscopic 3D tissue architecture, Adv. Mater. 23, (2011) H90-H94.

[37] S. Amemori, M. Matsusaki, M. Akashi, Biocompatible and highly sensitive nitric oxide sensor particles prepared by layer-by-layer assembly, Chem. Lett. 39, (2010) 42-43.

[38] P. Rajagopalan, C. J. Shen, F. Berthiaume, A. W. Tilles, M. Toner, M. L. Yarmush, Polyelectrolyte nano-scaffolds for the design of layered cellular architectures, Tissue Eng. 12 (2006) 1553-1563.

[39] K. Kadowaki, M. Matsusaki, M. Akashi, Three-dimensional constructs induce high cellular activity: structural stability and the specific production of proteins and cytokines, Biochem. Biophys. Res. Commun. 402, (2010) 153-157.

[40] K. Kadowaki, M. Matsusaki, M. Akashi, Control of cell surface and functions by layer-bylayer nanofilms, Langmuir 26, (2010) 5670-5678.

[41] M. Matsusaki, K. Kadowaki, E. Adachi, T. Sakura, U. Yokoyama, Y. Ishikawa, M. Akashi, Morphological and histological evaluations of 3D-layered blood vessel constructs prepared by hierarchical cell manipulation, J. Biomater. Sci. 23, (2012) 63-79.

[42] M. Matsusaki, S. Amemori, K. Kadowaki, M. Akashi, Quantitative 3D analysis of nitric oxide diffusion in a 3D artery model using sensor particles, Angew. Chem. Int. Ed. 50, (2011) 75577561. 
[43] W. K. Alderton, C. E. Cooper, R. G. Knowles, Nitric oxide synthases: structure, function and inhibition, Biochem. J. 357, (2001) 593-615.

[44] S. Kurioka, K. Koshimura, Y. Murakami, M. Nishiki, Y. Kato, Reverse correlation between urine nitric oxide metabolites and insulin resistance in patients with type 2 diabetes mellitus, Endocr. J. 47, (2000) 77-81.

[45] Y. Nakaya, A. Minami, N. Harada, S. Sakamoto, Y. Niwa, M. Ohnaka, Taurine improves insulin sensitivity in the otsuka long-evans tokushima fatty rat, a model of spontaneous type 2 diabetes, Am. J. Clin. Nutr. 71, (2000) 54-58.

[46] V. Mironov, T. Boland, T. Trusk, G. Forgacs, R. R. Markwald, Organ printing: computeraided jet-based 3D tissue engineering, TRENDS in Biotech. 21, (2003) 157-161.

[47] V. Mironov, V. Kasyanov, X. Z. Shu, C. Eisenberg, L. Eisenberg, S. Gonda, T. Trusk, R. R. Markwald, G. D. Prestwich, Fabrication of tubular tissue constructs by centrifugal casting of cells suspended in an in situ crosslinkable hyaluronan-gelatin hydrogel, Biomaterials 26, (2005) 7628-7635.

[48] Y. Nishiyama, C. Henmi, S. Iwanaga, H. Nakagawa, K. Yamaguchi, K. Akita, S. Mochizuki, K. Takiura, M. Nakamura, Ink jet three-dimensional digital fabrication for biological tissue manufacturing: analysis of alginate microgel beads produced by ink jet droplets for three dimensional tissue fabrication, Digital Fabrication 2006 (Society for Imaging Science and Technology, Springfield, VA, 2006), pp89-92.

[49] J. Folkman, C. C. Haudenschild, B. R. Zetter, Long-term culture of capillary endothelial cells, Proc. Natl. Acad. Sci. USA 76, (1979) 5217-5221.

[50] J. Folkman, C. Haudenschild, Angiogenesis in vitro, Nature 288, (1980) 551-556.

[51] R. E. Unger, A. Sartoris, K. Peters, A. Motta, C. Migliaresi, M. Kunkel, U. Bulnheim, J. Rychly, C. J. Kirkpatrick, Tissue-like self-assembly in cocultures of endothelial cells and osteoblasts and the formation of microcapillary-like structures on three-dimensional porous biomaterials, Biomaterials 28, (2007) 3965-3976. 
[52] S. Levenberg, J. Rouwkenma, M. Macdonald, E. S. Garfein, D. S. Kohane, D. C. Darland, R. Marini, C. A. van Blitterswijk, R. C. Mulligan, P. A. D’Amore, R. Langer, Engineering vascularized skeletal muscle tissue, Nat. Biotech. 23, (2005) 879-884.

[53] T. Sasagawa, T. Shimizu, S. Sekiya, Y. Haraguchi, M. Yamato, Y. Sawa, T. Okano, Design of prevascularized three-dimensional cell-dense tissues using a cell sheet stacking manipulation technology, Biomaterials 31, (2010) 1646-1654.

[54] Y. Zheng, J. Chen, M. Craven, N. W. Choi, S. Totorica, A. D.-Santana, P. Kermani, B. Hempstead, C. F.-Teschl, J. A. López, A. D. Stroock, In vitro microvessels for the study of angiogenesis and thrombosis, Proc. Natl. Acad. Sci. USA 109, (2012) 9342-9347.

[55] J. S. Miller, K. R. Stevens, M. T. Yang, B. M. Baker, D.-H. T. Nguyen, D. M. Cohen, E. Toro, A. A. Chen, P. A. Galie, X. Yu, R. Chaturvedi, S. N. Bhatia, C. S. Chen, Rapid casting of patterned vascular networks for prefusable engineered three-dimensional tissues, Nat. Mater. 11 (2012) 768-774.

[56] H. Sekine, T. Shimizu, K. Sakaguchi, I. Dobashi, M. Wada, M. Yamato, E. Kobayashi, M. Umezu, T. Okano, In vitro fabrication of functional three-dimensional tissues with perfusable blood vessels, Nat. Commun. (2013), DOI: 10.1038/ncomms2406.

[57] S. Kim, H. Lee, M. Chung, N. L. Jeon, Engineering of functional, perfusable 3D microvascular networks on a chip, Lab Chip, 13, (2013) 1489-1500.

[58] D.-H. T. Nguyen, S. C. Stapleton, M. T. Yang, S. S. Cha, C. K. Choi, P. A. Galie, C. S. Chen, Biomimetic model to reconstitute angiogenic sprouting morphogenesis in vitro, Proc. Natl. Acad. Sci. USA, 110, (2013) 6712-6717.

[59] P. Chetprayoon, K. Kadowaki, M. Matsusaki, M. Akashi, Survival and structural evaluations of three-dimensional tissues fabricated by the hierarchical cell manipulation technique, Acta Biomater. 9, (2013) 4698-4706.

[60] H. Yoshida, M. Matsusaki, M. Akashi, Multilayered blood capillary analogs in biodegradable hydrogels for in vitro drug permeability assays, Adv. Funct. Mater. 23, (2013) 1736-1742. 
[61] D. Curtis, A. Sood, T. Phillips, V. Leinster, A. Nishiguchi et al., unpublished data.

[62] G. Bhabra, A. Sood, B. Fisher, L. Cartwright, M. Saunders et al., Nanoparticles can cause DNA damage across a cellular barrier, Nat. Nanotech. 5, (2009) 876-883.

[63] A. Sood, S. Salih, D. Roh, L. Lacharme-Lora, M. Parry et al., Signalling of DNA damage and cytokines across cell barriers exposed to nanoparticles depends on barrier thickness, Nat. Nanotech. 6, (2011) 824-833.

[64] M. Matsusaki, K. Sakaue, K. Kadowaki, M. Akashi, Three-dimensional human tissue chips fabricated by rapid and automatic inkjet cell printing, Adv. Healthcare Maetr. 2, (2013) 534539.

[65] K. Takahashi, K. Tanabe, M. Ohnuki, M. Narita, T. Ichisaka, K. Tomoda, S. Yamanaka, Induction of pluripotent stem cells from adult human fibroblasts by defined factors, Cell 131, (2007) 861-872.

[66] M. Inamura, K. Kawabata, K. Takayama, K. Tashiro, F. Sakurai, K. katayama, M. Toyoda, H. Akutsu, Y, Miyagawa, H. Okita, N. Kiyokawa, A. Umezawa, T. Hayakawa, M. K. Furue, H. Mizuguchi, Efficient generation of hepatoblasts from human ES cells and iPS cells by transient overexpression of homeobox gene HEX, Mol. Ther. 19, (2011) 400-407. 


\section{Figure Captions}

Figure 1. Schematic illustration of the top-down and bottom-up approaches to fabricate 3D-tissue constructs.

Figure 2. Cartoon of the hierarchical cell manipulation technique by LbL assembly using FN and G. Reproduced with permission [34].

Figure 3. Histological images of 3D-tissue constructs. a) HE staining images of 1-, 2-, and 5LNHDFs. b) $\mathrm{HE}$ staining image of 3L-mouse $\mathrm{C} 2 \mathrm{C} 12$ myoblasts (top) and an immunostaining image with anti-factor VIII antibody for 4L-NHDF/1L-HUVEC structures (bottom). Reproduced with permission [36].

Figure 4. a) Cartoon of the cell accumulation technique using FN-G nanofilm fabrication. b) HE staining image of 23L-NHDF tissues after 24 hours of incubation. Reproduced with permission [37].

Figure 5. a) Schematic illustration of HUVEC tube formation by sandwich culture with NHDF tissues. b) Immunostaining image with anti-factor VIII antibody for 4L-NHDF-1LHUVEC-4L-NHDF tissues after 7 days of incubation. c) CLSM image of HUVEC and lymph endothelial cell (LEC) networks immunostained with anti-CD31 and anti-LYVE1 antibodies, respectively. Reproduced with permission [37].

Figure 6. a) Confocal fluorescent images of top (left) and cross-sectional (right) views of HUVECs fluorescently labeled with PKH26. b) Confocal fluorescent microscopic images of the bilayer morphology of cell tracker green-labeled UASMCs as the first layer, and the PKH26-labeled HUVECs as the second layer (top). The bottom image is a magnified 
image of the top. The scale bars are $5 \mathrm{~mm}$ in (a, left), $400 \mu \mathrm{m}$ in (a, right and b, top) and $100 \mu \mathrm{m}$ in (b, bottom). Reproduced with permission [58]. c) Schematic cartoon of the development of 3D micro-tissue arrays by the LbL inkjet printing of single cells, FN and G, respectively. Reproduced with permission [63]. 
Top-down approach

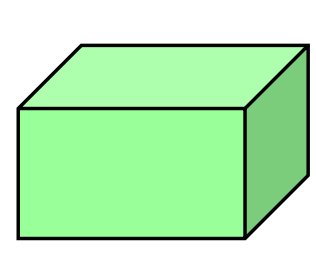

Hydrogels

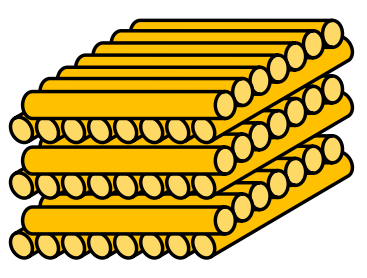

Fiber scaffolds

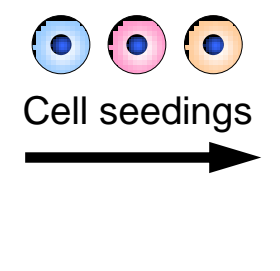

(n)

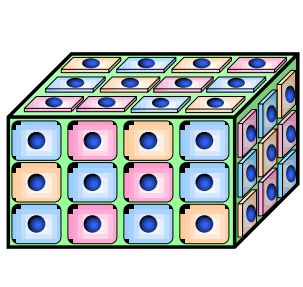

3D cell culture
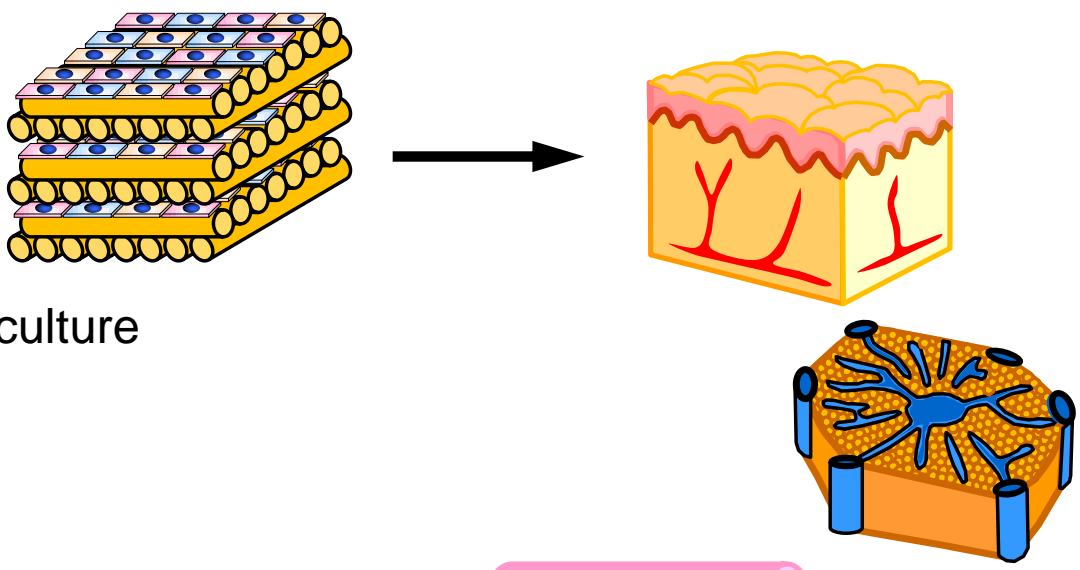

Bottom-up approach

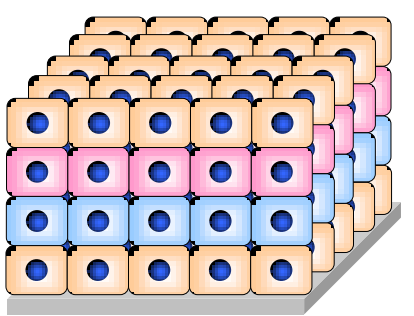

Cellular layer-by-layer
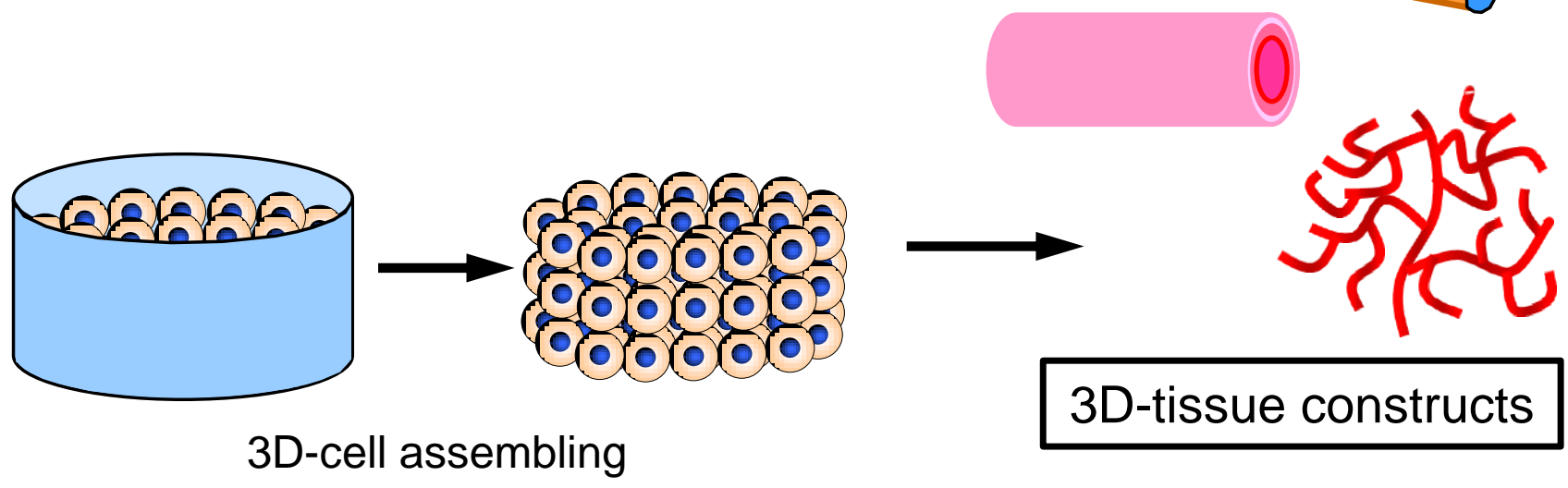

3D-tissue constructs 


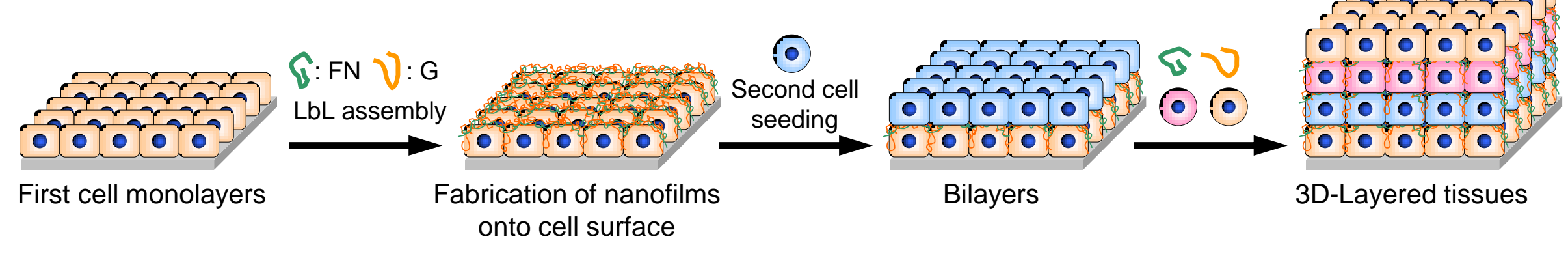

Figure 2. 
a)
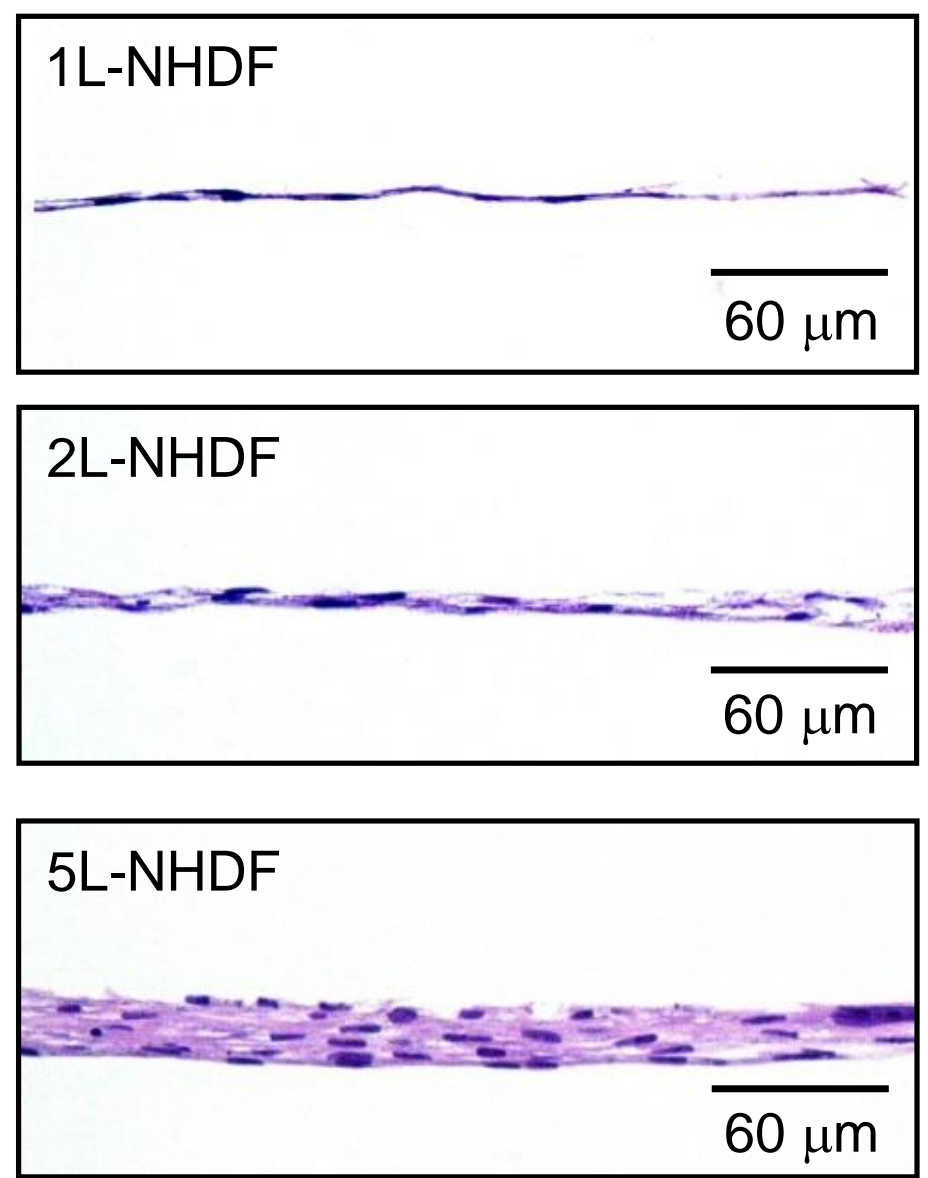

b)

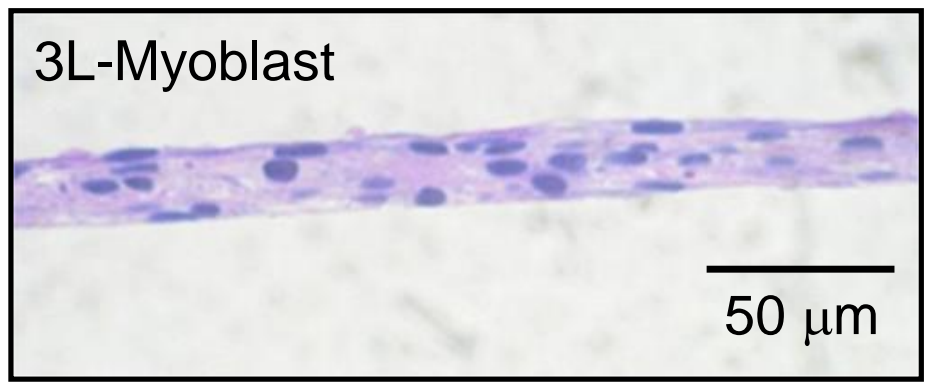

Factor IIIV 4L-NHDF/1L-HUVEC

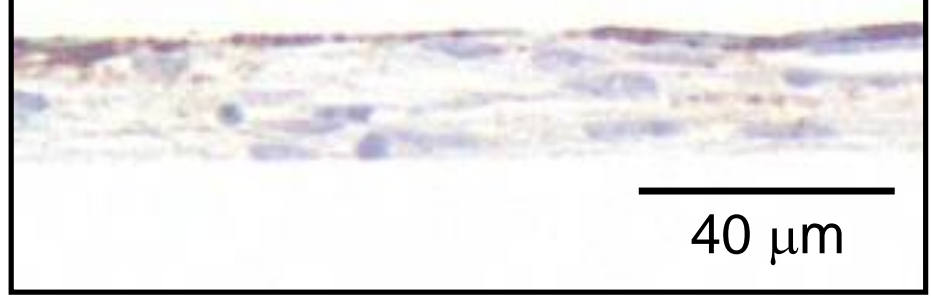

Figure 3. 
a)
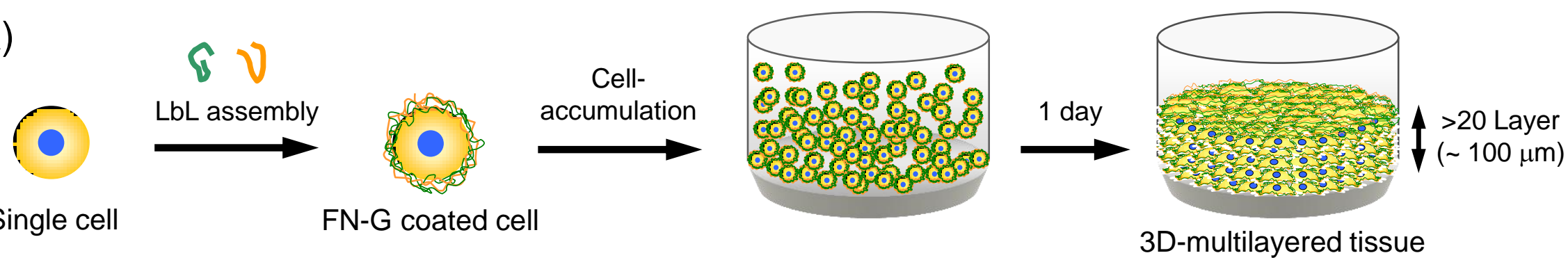

b) 23L-NHDF

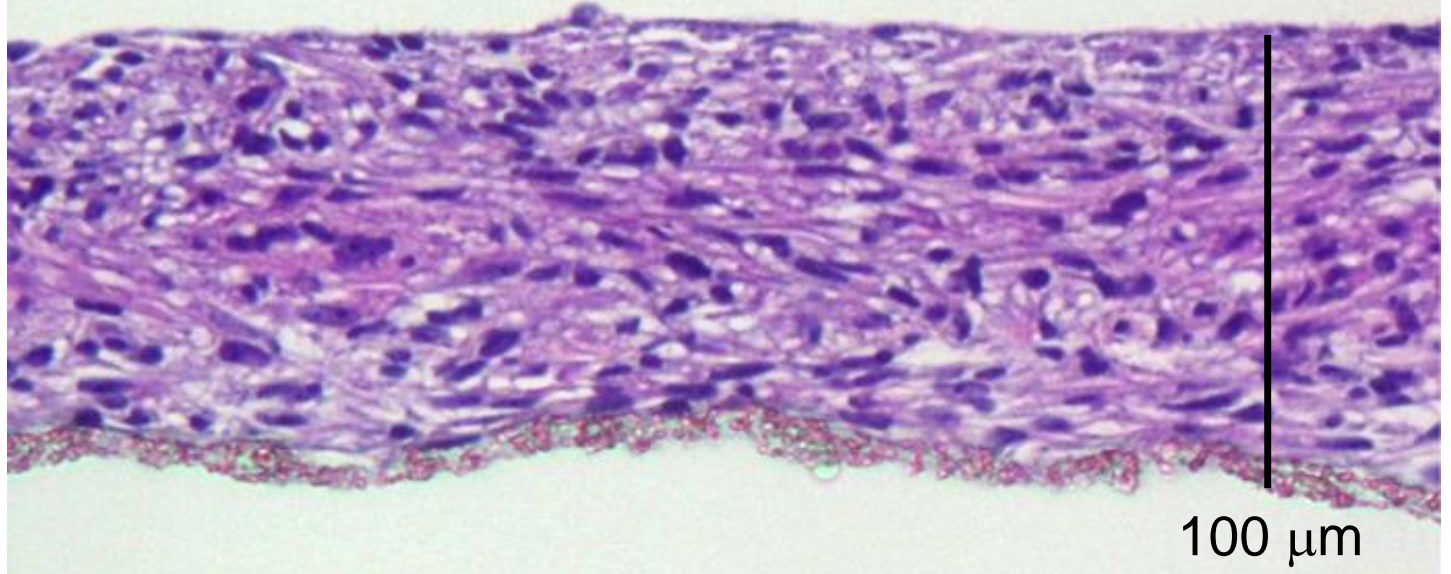

Figure 4. 
a)

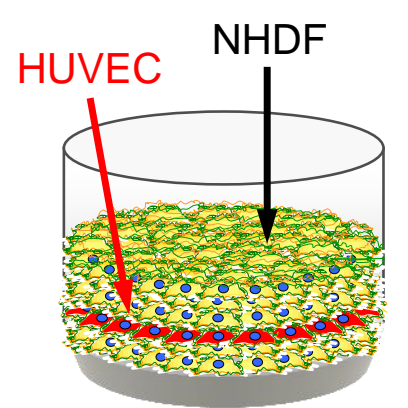

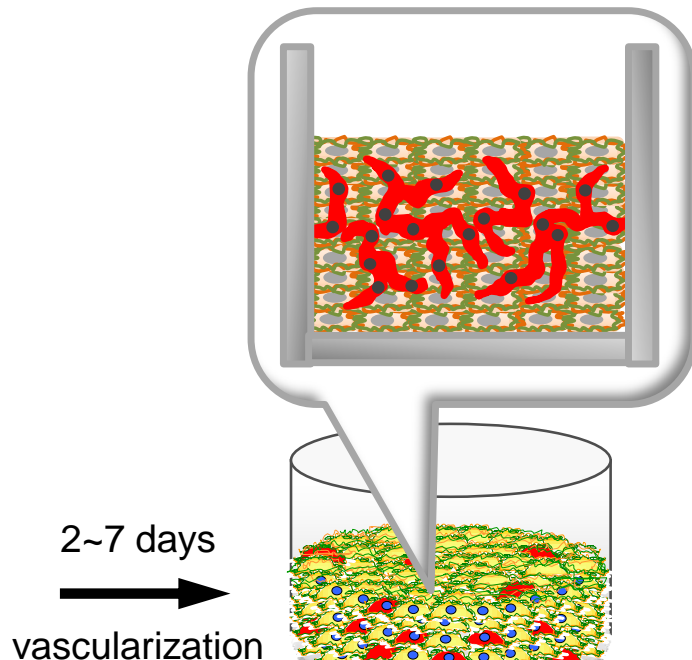

vascularization b) Factor VIII

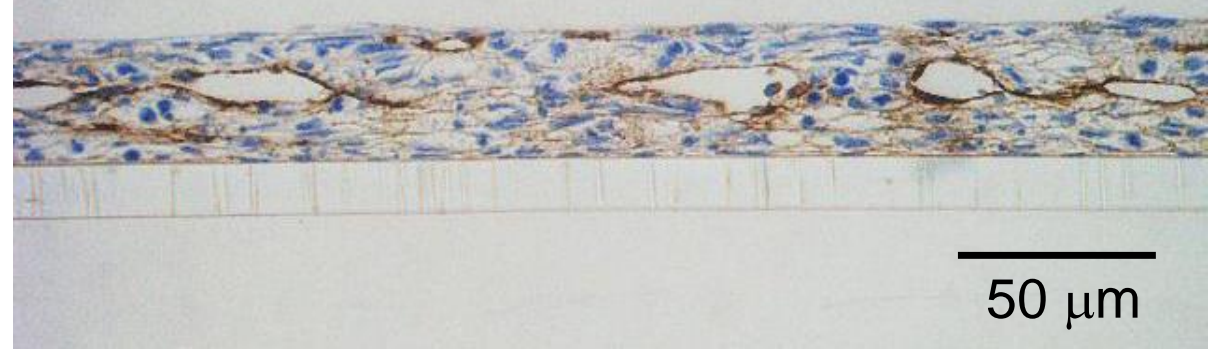

c)

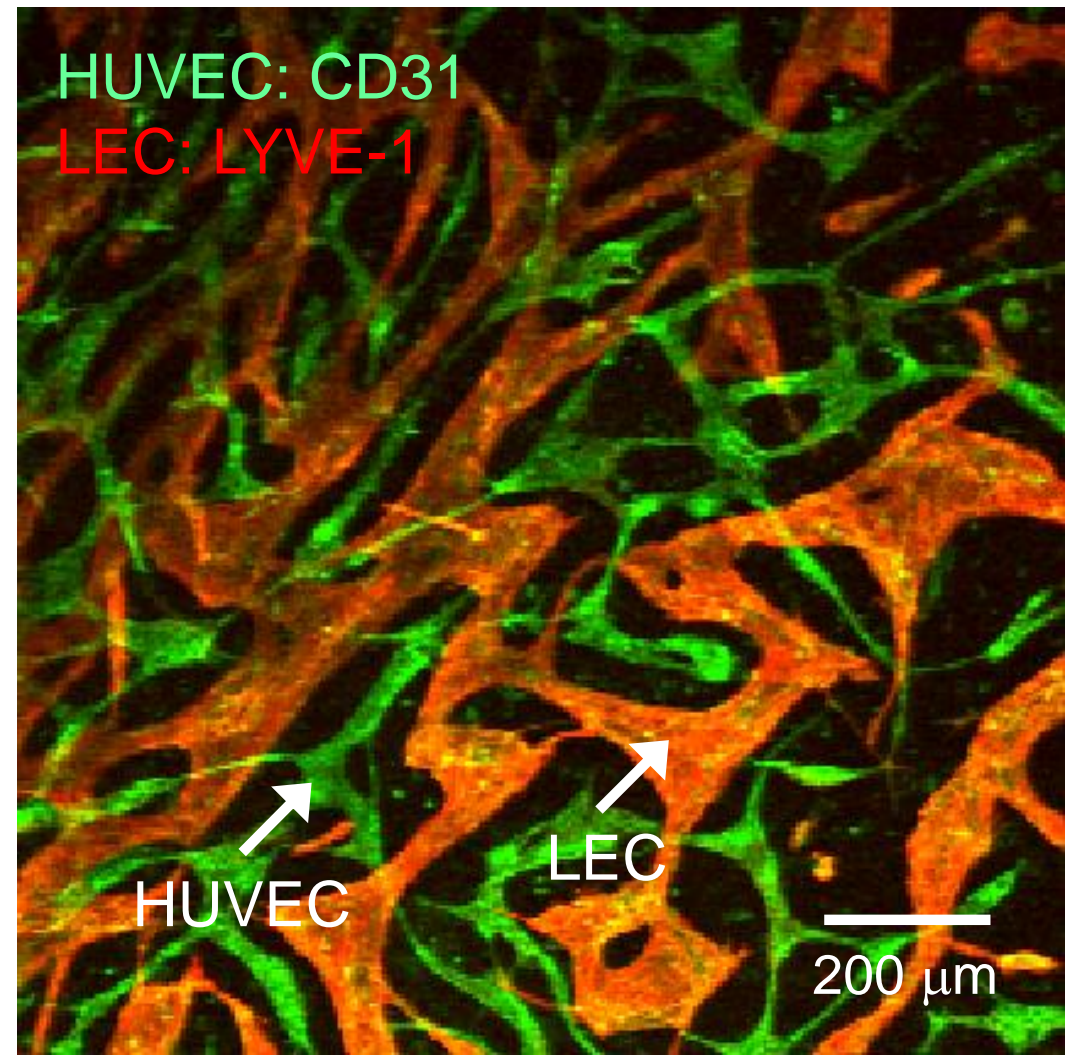

Figure 5. 
a)

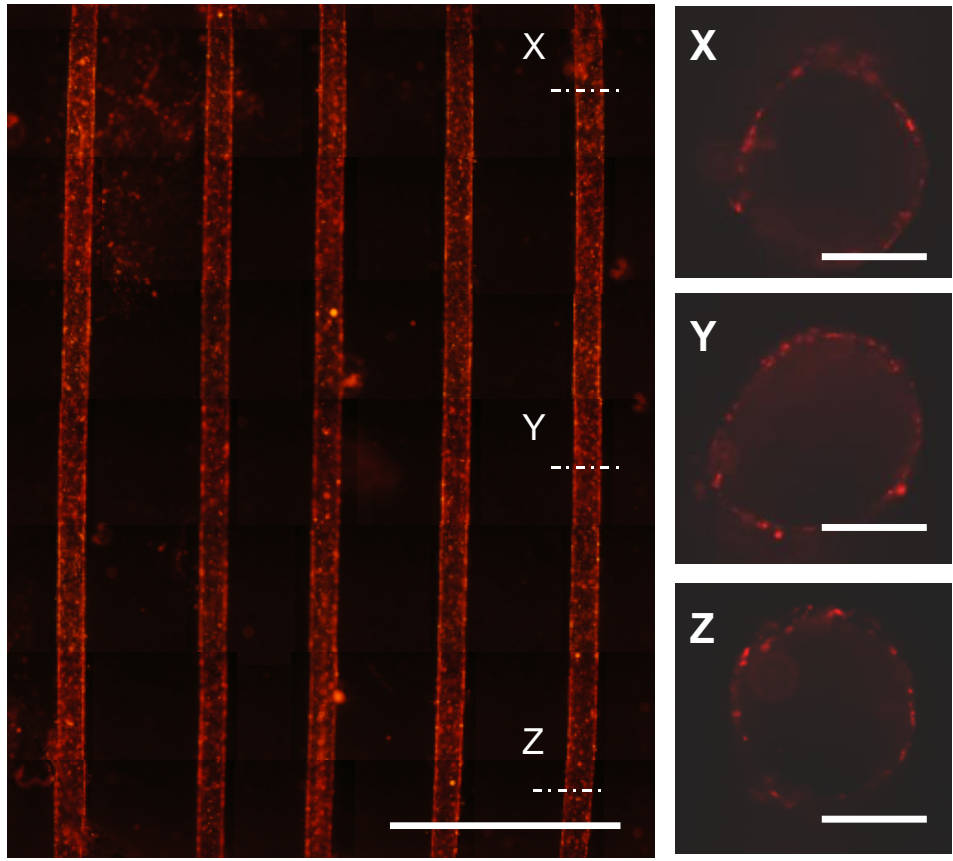

c)

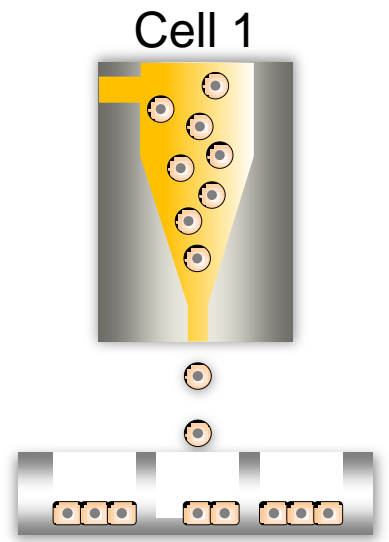

Cell printing for first layer

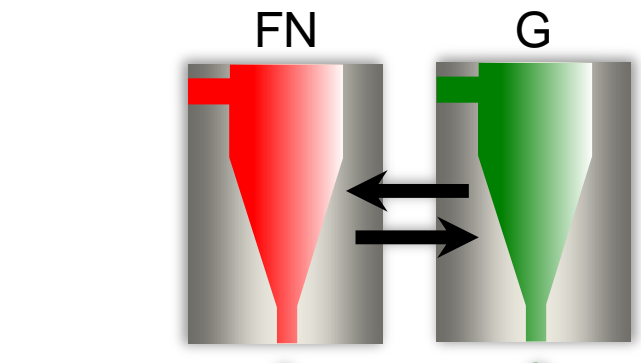

0

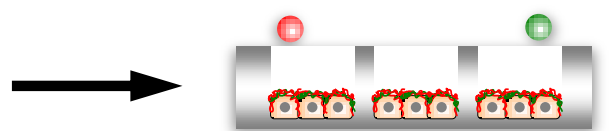

FN-G nanofilm preparation by alternating printing b)
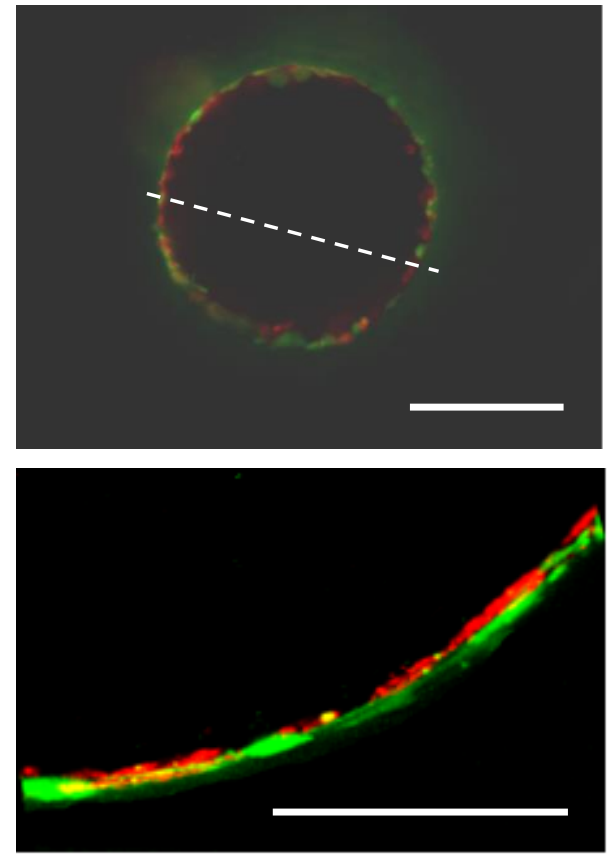

Cell 2

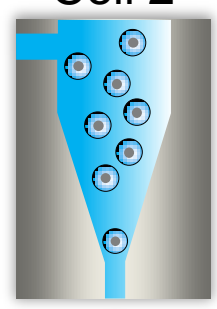

○

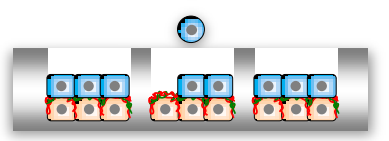

Repeating

Cell printing for second layer
Activity screening assays

1

ㅁํㅁำ

3D micro-tissue chips

Figure 6. 
